\title{
ON THE DYNAMICS OF A MODEL WITH COEXISTENCE OF THREE ATTRACTORS: A POINT, A PERIODIC ORBIT AND A STRANGE ATTRACTOR
}

\author{
JAUME LLIBRE AND CLAUDIA VALLS
}

\begin{abstract}
For a dynamical system described by a set of autonomous differential equations, an attractor can be either a point, or a periodic orbit, or even a strange attractor. Recently a new chaotic system with only one parameter has been presented where besides a point attractor and a chaotic attractor, it also has a coexisting attractor limit cycle which makes evident the complexity of such a system. We study using analytic tools the dynamics of such system. We describe its global dynamics at infinity, and show that it has no Darboux first integrals. Additionally, we characterize its Hopf bifurcations.
\end{abstract}

\section{INTRODUCTION AND STATEMENT OF THE MAIN RESULTS}

In this paper we study the differential system

$$
\begin{aligned}
& \dot{x}=a+y z, \\
& \dot{y}=-y+x^{2}, \\
& \dot{z}=1-4 x,
\end{aligned}
$$

where $a \in \mathbb{R}$ is a parameter, $x, y, z \in \mathbb{R}$ and the independent variable is the time $t$. This is probably the easiest known polynomial differential systems for which coexists at the same time for $a=1 / 100$ three attractors given by an equilibrium point, a periodic orbit and a strange attractor (see [8] for more details).

In general, to describe the global dynamics of a nonlinear differential system in $\mathbb{R}^{3}$ is a hard problem, usually unsolved. The goal of this paper is to analyze what can be said using analytical tools about the dynamics of system (1). We shall describe the global dynamics at infinity, we shall also study the Darboux integrability of the system, and finally we characterize its Hopf bifurcations.

The following result shows that the dynamics in a neighborhood of the infinity for system (1) does not depend on the parameter $a$. The study of the infinity for a polynomial differential system is made using the socalled Poincaré compactification, see section 2 for a brief introduction to such compactification.

Proposition 1. For all values of the parameter a the phase portrait of system (1) on the infinity of the Poincaré ball is topologically equivalent to

2010 Mathematics Subject Classification. Primary: 37D99.

Key words and phrases. Hopf bifurcation, chaotic system, Darboux integrability, Poincaré compactification. 


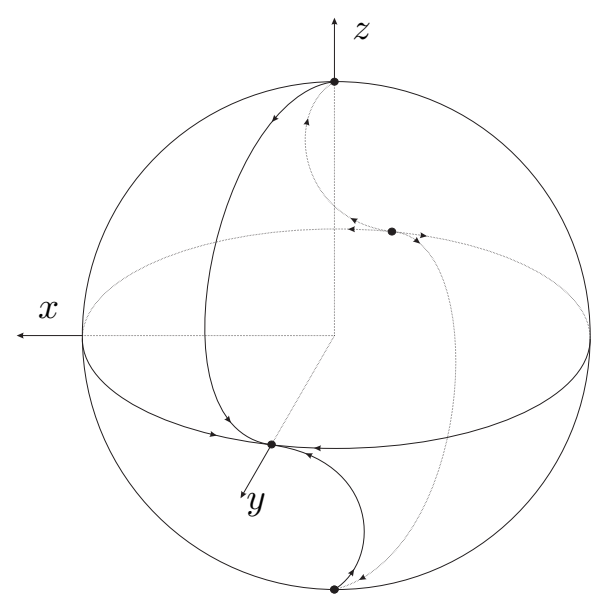

Figure 1. The phase portrait of system (1) on the infinity $\mathbb{S}^{2}$ of the Poincaré ball.

the one of Figure 1. There are two pairs of singular points at infinity. The pair which is at the endpoints of the $y$-axis are nodes, unstable the endpoint of the positive half-axis and stable the endpoint of the negative half-axis. The pair which is at the endpoints of the z-axis are nilpotent cusps.

Proposition 1 is proved in section 2 .

Let $\mathbb{C}[x, y, z]$ be the ring of the real polynomials in the variables $x, y$ and $z$. We say that $F=F(x, y, z) \in \mathbb{C}[x, y, z] \backslash\{0\}$ is a Darboux polynomial of system (1) if it satisfies

$$
(\nabla F) \cdot(P, Q, R)=k F,
$$

where $k=k(x, y z)$ is a real polynomial of degree at most 1 called the cofactor of $F(x, y z)$, and $\nabla F$ is the gradient of $F$. If the cofactor is zero, then $F(x, y, z)$ is a polynomial first integral of system (1). If $F(x, y, z)$ is a real Darboux polynomial with nonzero cofactor, then the surface $F(x, y, z)=0$ is an invariant algebraic surface, that is, if an orbit of system (1) has a point on this surface, then the whole orbit is contained in it.

The vector field associated to system (1) is

$$
\mathcal{X}=(a+y z) \frac{\partial}{\partial x}+\left(-y+x^{2}\right) \frac{\partial}{\partial y}+(1-4 x) \frac{\partial}{\partial z} .
$$

Let $U$ be an open subset of $\mathbb{R}^{3}$ such that $\mathbb{R}^{3} \backslash U$ has zero Lebesgue measure. We say that a non-constant real function $H=H(x, y, z): U \rightarrow \mathbb{R}$ is a first integral if $H(x(t), y(t), z(t))$ is constant on all solutions $(x(t), y(t), z(t))$ of $\mathcal{X}$ contained in $U$, i.e, $\left.\mathcal{X} H\right|_{U}=0$. The existence of a first integral for a differential system in $\mathbb{R}^{3}$ allows to reduce the study of the differential system in one dimension. This is the main reason to look for first integrals.

An exponential factor $F(x, y z)$ of the vector field $\mathcal{X}$ is an exponential function of the form $\exp (g / h)$ with $g$ and $h$ coprime polynomials in $\mathbb{C}[x, y, z]$, 
satisfying $\mathcal{X} F=L F$ for some $L \in \mathbb{C}[x, y, z]$ with degree one. The exponential factors appear when some Darboux polynomial has multiplicity larger than one, for more details see $[2,6]$.

A first integral of system (1) is called of Darboux type if its is a first integral of the form

$$
f_{1}^{\lambda_{1}} f_{2}^{\lambda_{2}} \cdots f_{p}^{\lambda_{p}} F_{1}^{\mu_{1}} \cdots F_{q}^{\mu_{q}}
$$

where $f_{1}, \ldots, f_{p}$ are Darboux polynomials and $F_{1}, \ldots, F_{q}$ are exponential factors.

Theorem 2. System (1) has no first integrals of Darboux type for all $a \in \mathbb{R}$.

The proof of Theorem 2 is given in section 3 .

In this paper we also characterize the Hopf bifurcations of system (1). The unique equilibrium point of system (1) is

$$
p=\left(\frac{1}{4}, \frac{1}{16},-16 a\right) .
$$

As we shall see later on the linearization of system (1) at $p_{0}$ has a pair of conjugate purely imaginary eigenvalues and one real eigenvalue for the unique value $a=0$ of the parameter $a$. Therefore when $a=0$ we have the setting for a Hopf bifurcation. That is, we can expect to have a smallamplitude limit cycle bifurcating from the equilibrium point $p_{0}$. But in order that this takes place it remains to compute the first Lyapunov coefficient $l_{1}\left(p_{0}\right)$ of system $(1)$ at the equilibrium $p_{0}$. When $l_{1}\left(p_{0}\right)<0$ the point $p_{0}$ is a weak focus of system (1) restricted to the central manifold and the limit cycle that emerges from $p_{0}$ is stable. In this case the Hopf bifurcation is called supercritical. When $l_{1}\left(p_{0}\right)>0$ the point $p_{0}$ is also a weak focus of system (1) restricted to the central manifold of $p$ but the limit cycle that born from $p_{0}$ is unstable. In this second case the Hopf bifurcation is called subcritical. For more details on the Hopf bifurcation see for instance the book of Kuznetsov see [5].

The next result characterize the Hopf bifurcation in system (1).

Theorem 3. System (1) has a subcritical Hopf bifurcation at the equilibrium point $p=\left(\frac{1}{4}, \frac{1}{16},-16 a\right)$ when $a=0$, and there exists a small $\varepsilon>0$ such that for $a \in(-\varepsilon, 0)$ the system has an unstable limit cycle.

Theorem 3 is proved in section 4 .

\section{The Poincaré COMPaCtification}

In what follows we present a summary on the Poicaré compactification of a polynomial vector field in $\mathbb{R}^{3}$, for more details see [3].

We consider the polynomial differential system

$$
\dot{x}=P^{1}(x, y, z), \quad \dot{y}=P^{2}(x, y, z), \quad z=P^{3}(x, y, z),
$$

in $\mathbb{R}^{3}$, or equivalently its associated polynomial vector field $X=\left(P^{1}, P^{2}, P^{3}\right)$. The degree $n$ of $X$ is defined as $n=\max \left\{\operatorname{deg}\left(P^{i}\right): i=1,2,3\right\}$.

Let $\mathbb{S}^{3}=\left\{y=\left(y_{1}, y_{2}, y_{3}, y_{4}\right) \in \mathbb{R}^{4}:\|y\|=1\right\}$ be the unit sphere in $\mathbb{R}^{4}$, and $\mathbb{S}_{+}=\left\{y \in \mathbb{S}^{3}: y_{4}>0\right\}$ and $\mathbb{S}_{-}=\left\{y \in \mathbb{S}^{3}: y_{4}<0\right\}$ be the northern 
and the southern hemispheres, respectively. We denote by $T_{y} \mathbb{S}^{3}$ the tangent space to $\mathbb{S}^{3}$ at the point $y$. We identify $\mathbb{R}^{3}$ with the tangent hyperplane

$$
T_{(0,0,0,1)} \mathbb{S}^{3}=\left\{\left(x_{1}, x_{2}, x_{3}, 1\right) \in \mathbb{R}^{4}:\left(x_{1}, x_{2}, x_{3}\right) \in \mathbb{R}^{3}\right\} .
$$

Doing central projections of the hyperplane $T_{(0,0,0,1)} \mathbb{S}^{3}$ on the sphere $\mathbb{S}^{3}$ we get two copies of our vector field $X$ on $\mathbb{S}^{3}$, one in the open nothern hemisphere $\mathbb{S}_{+}$and the other in the open southern hemisphere $\mathbb{S}_{-}$. Now the equator $\mathbb{S}^{2}=\mathbb{S}^{3} \cap\left\{y_{4}=0\right\}$ plays the role of the infinity of $\mathbb{R}^{3}$. There is a unique extension of the two copies of the polynomial vector field $X$ on $\mathbb{S}_{+} \cup \mathbb{S}_{-}$to an analytic vector field $p(X)$ on $\mathbb{S}^{3}$. This vector field $p(X)$ on $\mathbb{S}^{3}$ is called the Poincaré compactification of $X$.

Note that the projection of $\mathbb{S}_{+} \cup \mathbb{S}^{2}$ on the hyperplane $y_{4}=0$ through $\left(y_{1}, y_{2}, y_{3}, y_{4}\right) \mapsto\left(y_{1}, y_{2}, y_{3}\right)$ is the unit closed ball centered at the origin of $\mathbb{R}^{3}=\left\{\left(y_{1}, y_{2}, y_{3}\right)\right\}$. The interior of this ball is diffeomorphic to $\mathbb{R}^{3}$ and its boundary $\mathbb{S}^{2}$ corresponds to the infinity of $\mathbb{R}^{3}$. This ball is called the Poincaré ball.

We consider the following eight local charts on $\mathbb{S}^{3}$ :

$$
\begin{aligned}
U_{i} & =\left\{\left(y_{1}, y_{2}, y_{3}, y_{4}\right): y_{i}>0\right\}, \\
V_{i} & =\left\{\left(y_{1}, y_{2}, y_{3}, y_{4}\right): y_{i}<0\right\},
\end{aligned}
$$

for $i=1,2,3,4$. Then the analytical field $p(X)$ in the local chart $U_{1}$ becomes

$$
\frac{z_{3}^{n}}{(\Delta z)^{n-1}}\left(-z_{1} P^{1}+P^{2},-z_{2} P^{1}+P^{3},-z_{3} P^{1}\right)
$$

where $P^{i}=\left(1 / z_{3}, z_{1} / z_{3}, z_{2} / z_{3}\right)$.

In a similar way the expression of $p(X)$ in $U_{2}$ is

$$
\frac{z_{3}^{n}}{(\Delta z)^{n-1}}\left(-z_{1} P^{2}+P^{1},-z_{2} P^{2}+P^{3},-z_{3} P^{2}\right),
$$

where $P^{i}=P^{i}\left(z_{1} / z_{3}, 1 / z_{3}, z_{2} / z_{3}\right)$, and in $U_{3}$ is

$$
\frac{z_{3}^{n}}{(\Delta z)^{n-1}}\left(-z_{1} P^{3}+P^{1},-z_{2} P^{3}+P^{2},-z_{3} P^{3}\right),
$$

where $P^{i}=P^{i}\left(z_{1} / z_{3}, z_{2} / z_{3}, 1 / z_{3}\right)$.

In $U_{4}$ we have $z_{3}^{n+1}\left(P^{1}, P^{2}, P^{3}\right)$ in the expression for $p(X)$ where $P^{i}=$ $P^{i}\left(z_{1}, z_{2}, z_{3}\right)$. The expression for $p(X)$ in the local chart $V_{i}$ is the same as in $U_{i}$ multiplied by $(-1)^{n-1}$.

When we work with the expression of the compactified vector field $p(X)$ in the local charts we shall omit the common factor $1 /(\Delta z)^{n-1}$. We can do that through a rescaling of the time.

We remark that all the points on the sphere at infinity in the coordinates of any local chart have $z_{3}=0$.

In this section we study the behavior of the differential system (1) near the infinity using the Poincaré compactification. 
Proof of Proposition 1. From (3) the Poincaré compactification of system (1) in the local chart $U_{1}$ is

$$
\begin{aligned}
& \dot{z}_{1}=1-z_{1} z_{3}-z_{1}^{2} z_{2}-a z_{1} z_{3}^{2}, \\
& \dot{z}_{2}=-4 z_{3}+z_{3}^{2}-z_{1} z_{2}^{2}-a z_{2} z_{3}^{2}, \\
& \dot{z}_{3}=-z_{3}\left(z_{1} z_{2}+a z_{3}^{2}\right) .
\end{aligned}
$$

We look for the equilibria $\left(z_{1}, z_{2}, z_{3}\right)$ with $z_{3}=0$, which are the ones that are at infinity and we do not find any because the system in the local chart $U_{1}$ restricted to the infinity $z_{3}=0$ becomes

$$
\dot{z}_{1}=1-z_{1}^{2} z_{2}, \quad \dot{z}_{2}=-z_{1} z_{2}^{2} .
$$

So, the dynamics on $U_{1}$ is trivially conjugated to straight lines.

From (4) the Poincaré compactification of system (1) in the local chart $U_{2}$ is

$$
\begin{aligned}
& \dot{z}_{1}=z_{2}+z_{1} z_{3}+a z_{3}^{2}-z_{1}^{3}, \\
& \dot{z}_{2}=-4 z_{1} z_{3}+z_{2} z_{3}-z_{1}^{2} z_{2}+z_{3}^{2}, \\
& \dot{z}_{3}=-z_{3}\left(z_{1}^{2}-z_{3}\right) .
\end{aligned}
$$

This system with $z_{3}=0$ has a unique equilibrium point, the origin, i.e., the end-points of the positive $y$-axis. The system in the local chart $U_{2}$ restricted to $z_{3}=0$ writes

$$
\dot{z}_{1}=z_{2}-z_{1}^{3}, \quad \dot{z}_{2}=-z_{1}^{2} z_{2} .
$$

This system has the first integral

$$
H=\frac{2 z_{1}^{3}-3 z_{2}}{z_{2}^{3}} .
$$

Doing blow-ups we can study the local phase portrait at the origin $U_{2}$, obtaining a stable node, for more details on the blow-up change of variables see Chapters 2 and 3 of [4].

Finally, from (5) the Poincaré compactification of system (1) in the local chart $U_{3}$ is

$$
\begin{aligned}
& \dot{z}_{1}=z_{2}+a z_{3}^{2}+4 z_{1}^{2} z_{3}-z_{1} z_{3}^{2}, \\
& \dot{z}_{2}=z_{1}^{2}-z_{2} z_{3}+4 z_{1} z_{2} z_{3}-z_{2} z_{3}^{2}, \\
& \dot{z}_{3}=z_{3}^{2}\left(4 z_{1}-z_{3}\right) .
\end{aligned}
$$

Again the origin of this system with $z_{3}=0$ is the unique equilibrium point, i.e., the end-points of the positive $z$-axis. The system in the local chart $U_{3}$ restricted to $z_{3}=0$ writes

$$
\dot{z}_{1}=z_{2}, \quad \dot{z}_{2}=z_{1}^{2} .
$$

This system has the first integral

$$
H=2 z_{1}^{3}-3 z_{2}^{2} .
$$

The origin of $U_{3}$ is a nilpotent singular point. Using Theorem 3.15 of [4] its local phase portrait is a cusp. 


\section{Darboux integrability: Proof of Theorem 2}

To prove Theorem 2 first we state and show some auxiliary results.

Proposition 4. System (1) has no polynomial first integrals.

Proof. Let $f$ be a polynomial first integral and let $n \geq 1$ be its degree. Then the homogeneous part of degree $n$ of $f$ denoted by $f_{n}=f_{n}(x, y, z)$ satisfies the quasi-linear partial differential equation

$$
y z \frac{\partial f_{n}}{\partial x}+x^{2} \frac{\partial f_{n}}{\partial y}=0 .
$$

Its general solution is $F\left(z, 3 y^{2} z-2 x^{3}\right)$ being $F$ an arbitrary $C^{1}$ function. Since $f_{n}$ must be a homogeneous polynomial of degree $n$ we must have

$$
f_{n}=\sum_{j=0}^{n} a_{j} z^{n-3 j}\left(y^{2} z-2 x^{3}\right)^{j}, \quad a_{j} \in \mathbb{C} .
$$

Note that the homogeneous part of degree $n-1$ of $f$ denoted by $f_{n-1}=$ $f_{n-1}(x, y, z)$ satisfies

$$
y z \frac{\partial f_{n-1}}{\partial x}+x^{2} \frac{\partial f_{n-1}}{\partial y}-y \frac{\partial f_{n}}{\partial y}-4 x \frac{\partial f_{n}}{\partial z}=0
$$

that is

$$
\begin{aligned}
& y z \frac{\partial f_{n-1}}{\partial x}+x^{2} \frac{\partial f_{n-1}}{\partial y}-2 y^{2} z \sum_{j=0}^{n} j a_{j} z^{n-3 j}\left(y^{2} z-2 x^{3}\right)^{j-1} \\
& -4 x \sum_{j=0}^{n}(n-3 j) a_{j} z^{n-3 j-1}\left(y^{2} z-2 x^{3}\right)^{j} \\
& -4 x y^{2} \sum_{j=0}^{n} j a_{j} z^{n-3 j}\left(y^{2} z-2 x^{3}\right)^{j-1}=0 .
\end{aligned}
$$

We do the change of variables

$$
x=w, \quad y=\sqrt{\left(v+2 w^{3}\right) /(2 u)}, \quad z=u .
$$

In these variables equation (7) becomes

$$
\begin{aligned}
& \sqrt{\left(v+2 w^{3}\right) u / 2} \frac{d \tilde{f}_{n-1}}{d w}=\left(v+2 w^{3}\right) \sum_{j=0}^{n} j a_{j} u^{n-3 j} v^{j-1} \\
& -4 w \sum_{j=0}^{n}(n-3 j) a_{j} u^{n-3 j-1} v^{j}-2\left(v+2 w^{3}\right) w \sum_{j=0}^{n} j a_{j} u^{n-3 j-1} v^{j-1}
\end{aligned}
$$


where $\tilde{f}_{n-1}$ is $f_{n-1}$ written in the variables $u, v, w$. Integrating this equation we get

$$
\begin{aligned}
\tilde{f}_{n-1}= & \frac{\sqrt{2}}{105 \sqrt{u\left(2 w^{3}+v\right)}}\left(6 w(7 A-10 C w)\left(2 w^{3}+v\right)+\right. \\
& 30 \sqrt[3]{2} \sqrt[4]{3} v^{2 / 3}(14 B+3 C v) \sqrt{-\frac{(-1)^{5 / 6}\left(2 w^{3}+v\right)}{v}} \\
& E\left(\arcsin \left(\sqrt{-\frac{(-1)^{5 / 6}(\sqrt[3]{2} w+\sqrt[3]{v})}{\sqrt{3} \sqrt[3]{v}}}\right) \mid \sqrt[3]{-1}\right)+ \\
& \left.\sqrt[6]{-1} \sqrt[3]{2} 3^{3 / 4} v^{2 / 3}\right)\left(21 \sqrt[3]{2 v^{2 / 3} A+140(-1)^{2 / 3} B+}\right. \\
& \left.30(-1)^{2 / 3} C v\right) \sqrt{-\frac{(-1)^{5 / 6}\left(2 w^{3}+v\right)}{v}} \\
& F\left(\arcsin \left(\sqrt{-\frac{(-1)^{5 / 6}(\sqrt[3]{2} w+\sqrt[3]{v})}{\sqrt{3} \sqrt[3]{v}}}\right) \mid \sqrt[3]{-1}\right)
\end{aligned}
$$

where $F(\Phi \mid m)$ and $E(\Phi \mid m)$ are the elliptic integral of the first and second kind, respectively, for more details see [1]; and

$$
\begin{aligned}
A & =\sum_{j=0}^{n} j a_{j} u^{n-3 j} v^{j-1}, \\
B & =\sum_{j=0}^{n}(n-3 j) a_{j} u^{n-3 j-1} v^{j}, \\
C & =\sum_{j=0}^{n} j a_{j} u^{n-3 j-1} v^{j-1} .
\end{aligned}
$$

Now introducing the inverse change of (8), i.e.

$$
u=z, \quad v=3 y^{2} z-2 x^{3}, \quad w=x,
$$

we obtain

$$
\begin{aligned}
f_{n-1}= & \frac{\sqrt{2}}{105 \sqrt{3} y z}\left(18 x(7 A-10 C x) z y^{2}-\right. \\
& 30 \sqrt[3]{2} 3^{3 / 4} \sqrt{\frac{(-1)^{5 / 6} y^{2} z}{2 x^{3}-3 y^{2} z}}\left(3 y^{2} z-2 x^{3}\right)^{2 / 3}\left(6 C x^{3}-14 B-9 C y^{2} z\right) \\
& E\left(\arcsin \left(\sqrt{-\frac{(-1)^{5 / 6}\left(\sqrt[3]{2} x+\sqrt[3]{3 y^{2} z-2 x^{3}}\right)}{\sqrt{3}} \sqrt[3]{3 y^{2} z-2 x^{3}}}\right) \mid \sqrt[3]{-1}\right)+ \\
& 3 \sqrt[6]{-1} \sqrt[3]{2} \sqrt[4]{3} \sqrt{\frac{(-1)^{5 / 6} y^{2} z}{2 x^{3}-3 y^{2} z}}\left(3 y^{2} z-2 x^{3}\right)^{2 / 3}\left(21 \sqrt[3]{2}\left(3 y^{2} z-2 x^{3}\right)^{2 / 3} A+\right. \\
& \left.140(-1)^{2 / 3} B+30(-1)^{2 / 3} C\left(3 y^{2} z-2 x^{3}\right)\right) \\
& F\left(\arcsin \left(\sqrt{-\frac{(-1)^{5 / 6}\left(\sqrt[3]{2} x+\sqrt[3]{3 y^{2} z-2 x^{3}}\right)}{\sqrt{3} \sqrt[3]{3 y^{2} z-2 x^{3}}}}\right) \mid \sqrt[3]{-1}\right),
\end{aligned}
$$


where

$$
\begin{aligned}
& A=\sum_{j=0}^{n} j a_{j} z^{n-3 j}\left(3 y^{2} z-2 x^{3}\right)^{j-1}, \\
& B=\sum_{j=0}^{n}(n-3 j) a_{j} z^{n-3 j-1}\left(3 y^{2} z-2 x^{3}\right)^{j}, \\
& C=\sum_{j=0}^{n} j a_{j} z^{n-3 j-1}\left(3 y^{2} z-2 x^{3}\right)^{j-1} .
\end{aligned}
$$

Since $f_{n-1}$ must be a homogeneous polynomial of degree $n-1$, we must have

$$
\begin{aligned}
& 6 C x^{3}-14 B-9 C y^{2} z=-14 B+C\left(6 x^{3}-9 y^{2} z\right) \\
& =-14 \sum_{j=0}^{n}(n-3 j) a_{j} z^{n-3 j-1}\left(y^{2} z-2 x^{3}\right)^{j}+\left(6 x^{3}-9 y^{2} z\right) \sum_{j=0}^{n} j a_{j} u^{n-3 j-1} v^{j-1}=0
\end{aligned}
$$

and

$$
\begin{aligned}
& \left.21 \sqrt[3]{2}\left(3 y^{2} z-2 x^{3}\right)^{2 / 3} A+140(-1)^{2 / 3} B+30(-1)^{2 / 3} C\left(3 y^{2} z-2 x^{3}\right)\right) \\
& =21 \sqrt[3]{2}\left(3 y^{2} z-2 x^{3}\right)^{2 / 3} \sum_{j=0}^{n} j a_{j} u^{n-3 j} v^{j-1} \\
& \quad+140(-1)^{2 / 3} \sum_{j=0}^{n}(n-3 j) a_{j} z^{n-3 j-1}\left(y^{2} z-2 x^{3}\right)^{j} \\
& \quad+30(-1)^{2 / 3}\left(3 y^{2} z-2 x^{3}\right) \sum_{j=0}^{n} j a_{j} u^{n-3 j-1} v^{j-1}=0 .
\end{aligned}
$$

Clearly, from (10) we get that

$$
\sum_{j=0}^{n} j a_{j} u^{n-3 j} v^{j-1}=0,
$$

which implies that $a_{j}=0$ for $j=1, \ldots, n$. So, equation (9) becomes

$$
-14 n a_{0} z^{n-1}=0,
$$

which yields $a_{0}=0$. So, $a_{j}=0$ for $j=0, \ldots, n$ and thus $f_{n}=0$ in contradiction with the fact that $f_{n}$ is a polynomial of degree $n \geq 1$. This concludes the proof of the proposition.

Proposition 5. System (1) has no Darboux polynomials with non-zero cofactor.

Proof. Let $f(x, y, z)$ be a Darboux polynomial of system (1) with nonzero cofactor $k=k_{0}+k_{1} x+k_{2} y+k_{3} z$. Clearly, if follows from (2) that $f$ satisfies

$$
(a+y z) \frac{\partial f}{\partial x}+\left(-y+x^{2}\right) \frac{\partial f}{\partial y}+(1-4 x) \frac{\partial f}{\partial z}=k f .
$$


We expand $f$ as a power series in the variable $z$ and let $m$ be the degree of the polynomial in the variable $z$, so

$$
f=\sum_{j=0}^{m} f_{j}(x, y) z^{j}
$$

The terms of degree $m+1$ in $z$ in equation (11) satisfy

$$
y \frac{\partial f_{m}}{\partial x}=k_{3} f_{m}, \quad \text { that is } f_{m}=K_{m}(y) \exp \left(k_{3} x / y\right),
$$

where $K_{m}(y)$ is a $C^{1}$-function in the variable $y$. Since $f_{m}$ must be a polynomial we get that either $k_{3}=0$ and $K_{m}(y)$ is a polynomial in the variable $y$, or if $k_{3} \neq 0$ then $f_{m}=0$. In this last case $f=f(x, y)$ and satisfies

$$
(a+y z) \frac{\partial f}{\partial x}+\left(-y+x^{2}\right) \frac{\partial f}{\partial y}=\left(k_{0}+k_{1} x+k_{2} y+k_{3} z\right) f .
$$

Since $f$ does not depend on $z$, in particular (12) must hold for $z=0$ and so

$$
a \frac{\partial f}{\partial x}+\left(-y+x^{2}\right) \frac{\partial f}{\partial y}=\left(k_{0}+k_{1} x+k_{2} y\right) f .
$$

Solving (13) we get that if $a \neq 0$ then

$$
f(x, y)=\exp \left(\frac{3 x\left(2 k_{0}+k_{1} x\right)+2 k_{2}\left(6 a^{3}+x^{3}-3 a y\right)}{6 a}\right) K_{0}\left(\exp (x / a)\left(-2 a^{2}+2 a x-x^{2}+y\right)\right),
$$

and if $a=0$,

$$
f(x, y)=\exp \left(-k_{2} y\right)\left(y-x^{2}\right)^{k_{0}-x\left(k_{1}+k_{2} x\right)} K_{0}(x),
$$

where $K_{0}$ is a $C^{1}$-function in its variables.

When $a \neq 0, f(x, y)$ is never a polynomial unless $k_{0}=k_{1}=k_{2}=0$ and $K_{0}$ is constant, but then $f(x, y)$ is constant, in contradiction with the fact that $f$ is a Darboux polynomial.

When $a=0$ in order that $f$ is a polynomial we must have $k_{1}=k_{2}=0$ and then $f(x, y)=\left(y-x^{2}\right)^{-k_{0}} p(x)$ where $k_{0} \in \mathbb{N}^{-}$and $p(x) \in \mathbb{C}[x]$. Imposing that $f$ must satisfy (12) we get that

$$
k_{3}\left(x^{2}-y\right) p(x)+2 k_{0} x y p(x)+\left(y^{2}-x^{2} y\right) p^{\prime}(x)=0,
$$

which yields

$$
p(x)=c_{0} \exp \left(\frac{k_{3} x}{y}\right)\left(x^{2}-y\right)^{k_{0}},
$$

where $c_{0}$ is a constant. Since $k_{3} \neq 0$ we get a contradiction with the fact that $p(x)$ must be a polynomial, so this case is also not possible.

In short, $k_{3}=0$. Now we expand $f$ as a power series in the variable $y$ and let $\ell$ be the degree of the polynomial in the variable $y$, so

$$
f=\sum_{j=0}^{\ell} f_{j}(x, z) y^{j} .
$$

The terms of degree $\ell+1$ in $y$ in equation (11) satisfy

$$
z \frac{\partial f_{\ell}}{\partial x}=k_{2} f_{\ell}, \quad \text { that is } \quad f_{\ell}=K_{\ell}(z) \exp \left(k_{2} x / z\right),
$$


where $K_{\ell}(z)$ is a $C^{1}$-function in the variable $z$. Since $f_{\ell}$ must be a polynomial we get that either $k_{2}=0$ and $K_{\ell}(z)$ is a polynomial in the variable $z$, or if $k_{2} \neq 0$ then $f_{\ell}=0$. In this last case $f=f(x, z)$ and satisfies

$$
(a+y z) \frac{\partial f}{\partial x}+(1-4 x) \frac{\partial f}{\partial z}=\left(k_{0}+k_{1} x+k_{2} y\right) f .
$$

Solving (14) we get

$$
\begin{aligned}
f= & \exp \left(\frac{1}{8 y^{3 / 2}}\left(2 \sqrt{y}\left(k_{1}(a+y z)\right)-y\left(4 k_{0}+k_{1}+4 k_{2} y\right) \arctan \left(\frac{(4 x-1) \sqrt{y}}{2(a+y z)}\right)\right)\right. \\
& K_{0}\left(x(-1+2 x)+a z+y z^{2} / 2\right)
\end{aligned}
$$

where $K_{0}$ is a $C^{1}$ function in its variable. Since $f$ must be a polynomial we get that $k_{0}=k_{1}=k_{2}=0$ in contradiction with the fact that $k_{2} \neq 0$. So, $k_{2}=0$ and $k=k_{0}+k_{1} x$.

Now let $n$ be the degree of the polynomial $f$. Then the homogeneous part of degree $n$ of $f$ denoted by $f_{n}=f_{n}(x, y, z)$ satisfies

$$
y z \frac{\partial f_{n}}{\partial x}+x^{2} \frac{\partial f_{n}}{\partial y}=k_{1} x f_{n}
$$

Solving this partial differential equation we obtain

$f_{m}=K_{n}\left(z, 3 y^{2} z-2 x^{3}\right) \exp \left(-\frac{\sqrt{3} k_{1} x^{2} y \sqrt{\frac{y^{2} z}{3 y^{2} z-2 x^{3}}} 2 F_{1}\left(\frac{2}{3}, \frac{1}{2} ; \frac{5}{3} ; \frac{2 x^{3}}{2 x^{3}-3 y^{2} z}\right)}{2 y^{2} z}\right)$,

where $K_{n}\left(z, 3 y^{2} z-2 x^{3}\right)$ is a $C^{1}$ function in the variables $z$ and $3 y^{2} z-$ $2 x^{3}$, and ${ }_{2} F_{1}$ is the hypergeometric function, see [1]. Since $f_{n}$ must be a homogeneous polynomial of degree $n \geq 1$ (otherwise $f$ would not be a Darboux polynomial) we must have $k_{1}=0$, then $k=k_{0}$ and also

$$
f_{n}=K_{n}\left(z, 3 y^{2} z-2 x^{3}\right)=\sum_{j=0}^{n} a_{j} z^{n-3 j}\left(y^{2} z-2 x^{3}\right)^{j}, \quad a_{j} \in \mathbb{C} .
$$

Now proceeding as in the proof of Proposition 4 we get that in order that $f$ be a Darboux polynomial $k_{0}$ must be 0 . But this is not possible because $k=k_{0} \neq 0$. This concludes the proof.

Before proving Theorem 2 we also recall the following two well-known results of the Darboux theory of integrability. For the first one see for instance Chapter 8 of [4], and for the second one see [6, 7].

Theorem 6 (Darboux theory of integrability). Suppose that a polynomial vector field $\mathcal{X}$ defined in $\mathbb{R}^{n}$ of degree $m$ admits $p$ Darboux polynomials $f_{i}$ with cofactors $K_{i}$ for $i=1, \ldots, p$, and $q$ exponential factors $F_{j}=\exp \left(g_{j} / h_{j}\right)$ with cofactors $L_{j}$ for $j=1, \ldots, q$. If there exist $\lambda_{i}, \mu_{j} \in \mathbb{C}$ not all zero such that

$$
\sum_{i=1}^{p} \lambda_{i} K_{i}+\sum_{j=1}^{q} \mu_{j} L_{j}=0
$$

then the following real (multivalued) function of Darboux type

$$
f_{1}^{\lambda_{1}} \cdots f_{p}^{\lambda_{p}} F_{1}^{\mu_{1}} \cdots F_{q}^{\mu_{q}}
$$


substituting $f_{i}^{\lambda_{i}}$ by $\left|f_{i}\right|^{\lambda_{i}}$ if $\lambda_{i} \in \mathbb{R}$, is a first integral of the vector field $\mathcal{X}$.

Proposition 7. The following statements hold.

(1) If $e^{g / h}$ is an exponential factor for the polynomial differential system (1) and $h$ is a non-constant real polynomial, then $h=0$ is an invariant algebraic surface.

(2) Eventually $e^{g}$ can be an exponential factor, coming from the multiplicity of the infinite invariant plane.

Proof of Theorem 2. It follows from Propositions 7, 4 and 5 that if system (1) has an exponential factor then it must be of the form $e^{g}$ with $g \in$ $\mathbb{C}[x, y, z]$. Then $g$ must satisfy

$$
(a+y z) \frac{\partial g}{\partial x}+\left(-y+x^{2}\right) \frac{\partial g}{\partial y}+(1-4 x) \frac{\partial g}{\partial z}=L,
$$

where $L=l_{0}+l_{1} x+l_{2} y+l_{3} z$. Let $n$ be the degree of $g$. If $n \geq 2$ then if we write $g$ in its homogeneous parts as $g=\sum_{j=0}^{n} g_{j}(x, y, z)$ where $g_{j}=$ $g_{j}(x, y, z)$ is a homogeneous polynomial in the variables $x, y, z$ we have that $g_{n}$ satisfies (6). Proceeding as in the proof of Proposition 4 we obtain that $g_{n}=0$. Therefore, $g$ has degree one, that is, $g=g_{0}+g_{1} x+g_{2} y+g_{3} z$. Introducing $g$ in (15) we get that $g=c_{0}+c_{1} z$ with $L=c_{1}(1-4 x), c_{0}, c_{1} \in \mathbb{C}$. Now Theorem 6 implies that system (1) has no first integrals of Darboux type.

\section{Hopf BIFURCATION}

In order to prove that system (1) exhibits a Hopf bifurcation for the unique value $a$ of its parameter $a$, we will use the following theorem (see [5], page 178) which shows how to compute the first Lyapunov constant $l_{1}(p)$ at the equilibrium point $p$. When $l_{1}(p)<0$ the point $p$ is a weak focus of the system restricted to the central manifold of $p$ and the limit cycle that emerges from $p$ is stable. In this case we say that the Hopf bifurcation is supercritical. When $l_{1}(p)>0$ the point $p$ is also a weak focus of the system restricted to the central manifold of $p$ but the limit cycle that born from $p$ is unstable. In this case we say that the Hopf bifurcation is subcritical.

Theorem 8. Assume that a differential system $\dot{\mathbf{x}}=F(\mathbf{x})$ has $p$ as an equilibrium point and that the third order Taylor expansion of the function $F$ around the equilibrium $p$ is

$$
F(\mathbf{x})=A \mathbf{x}+\frac{1}{2 !} B(\mathbf{x}, \mathbf{x})+\frac{1}{3 !} C(\mathbf{x}, \mathbf{x}, \mathbf{x})+O\left(|x|^{4}\right) .
$$

Suppose that $A$ has a pair $\pm \lambda i$ of purely imaginary eigenvalues, and that $q$ is the eigenvector of $A$ with eigenvalue $\lambda i$ satisfying $q \bar{q}=1$, where $\bar{q}$ is the conjugate vector of $q$. Let $p$ be the eigenvector of $A^{T}$ with eigenvalue $-\lambda i$ satisfying $\bar{p} q=1$. Let $I$ be the identity matrix. Then the first Liapunov constant $l_{1}(p)$ of the differential system $\dot{\mathbf{x}}=F(\mathbf{x})$ at the equilibrium point $p$ is

$\frac{1}{2 \lambda} \operatorname{Re}\left(\bar{p} \cdot C(q, q, \bar{q})-2 \bar{p} \cdot B\left(q, A^{-1} B(q, \bar{q})\right)+\bar{p} \cdot B\left(\bar{q},(2 \lambda i I-A)^{-1} B(q, q)\right)\right)$. 
Proof of Theorem 3. The point $p=(1 / 4,1 / 16,-16 a)$ is an equilibrium of system (1). We translate the equilibrium at the origin with the following coordinate change.

$$
x=X+\frac{1}{4}, \quad y=Y+\frac{1}{16}, \quad z=Z-16 a,
$$

In the new coordinates, again denoted by $(x, y, z)$ instead of $(X, Y, Z)$ system (1) becomes

$$
\begin{aligned}
\dot{x} & =-16 a y+\frac{z}{16}+y z, \\
\dot{y} & =\frac{x}{2}-y+x^{2}, \\
\dot{z} & =-4 x
\end{aligned}
$$

The linear part of system (17) at the equilibrium point $(0,0,0)$ is given by the matrix

$$
A=\left(\begin{array}{ccc}
0 & -16 a & \frac{1}{16} \\
\frac{1}{2} & -1 & 0 \\
-4 & 0 & 0 .
\end{array}\right)
$$

The characteristic polynomial of this matrix is

$$
p(w)=-\frac{1}{4}-\left(\frac{1}{4}+8 a\right) w-w^{2}-w^{3} .
$$

Writing the polynomial

$$
p(w)=(w-\rho)(w-\varepsilon-i \beta)(w-\varepsilon+i \beta),
$$

we get

$$
\rho=-1-2 \varepsilon, \quad \beta=\frac{\sqrt{1-4 \varepsilon^{2}-8 \varepsilon^{3}}}{2 \sqrt{1+2 \varepsilon}}, \quad a=-\frac{\varepsilon\left(5+16 \varepsilon+16 \varepsilon^{2}\right)}{16(1+2 \varepsilon)} .
$$

We change the parameter $a$ by the parameter $\varepsilon$ by doing

$$
a=-\frac{\varepsilon\left(5+16 \varepsilon+16 \varepsilon^{2}\right)}{16(1+2 \varepsilon)} .
$$

In order that a Hopf bifurcation can take place at the origin of the differential system (17) $\varepsilon$ must be zero because then the eigenvalues at $p$ are $-1, \pm i / 2$. So, a Hopf bifurcation will take at the origin $O$ when $\varepsilon=0$ (i..e, $a=0$ ) if the Lyapunov constant $l_{1}(O)$ is not zero. We compute $l_{1}(O)$ using Theorem 8 . We need to write the function

$$
F(\mathbf{x})=F(x, y, z)=\left(F_{1}(x, y, z), F_{2}(x, y, z), F_{3}(x, y, z)\right)
$$

of the differential system (17) as it appears in (16). We already now the matrix $A$, then an easy computation shows that the bilinear form $B(\mathbf{x}, \mathbf{y})$ of (16) for our system (17) is

$$
B((x, y, z),(u, v, w))=(w y+v z, 2 u x, 0) .
$$

Since the polynomial differential system (17) is quadratic it follows that the trilinear form $C(\mathbf{x}, \mathbf{y}, \mathbf{z})$ of (16) is zero. 
Computing the normalized eigenvector $q$ of $A$, associated to the eigenvalue $\lambda i=i / 2$ we obtain

$$
q=\left(-\frac{i}{8},-\frac{1}{40}-\frac{i}{20}, 1\right)^{T}
$$

The normalized adjoint eigenvector of the transpose matrix $A$ with the eigenvalue $-\lambda i=-i / 2$ is

$$
p=\left(-i \sqrt{\frac{163}{10}}, 0, \frac{1}{8} \sqrt{\frac{163}{10}}\right)^{T}
$$

From Theorem 8 in order to compute $l_{1}(O)$ we must compute the quantities $-2 \bar{p} \cdot B\left(q, A^{-1} B(q, \bar{q})\right)$ and $\bar{p} \cdot B\left(\bar{q},(2 \lambda i I-A)^{-1} B(q, q)\right)$. We have

$$
-2 \bar{p} \cdot B\left(q, A^{-1} B(q, q)\right)=\frac{256}{815}+i \frac{72}{815}
$$

and

$$
\bar{p} \cdot B\left(\bar{q},(2 \lambda i I-A)^{-1} B(q, q)\right)=-\frac{42}{163}+i \frac{2}{489} .
$$

Then

$l_{1}(O)=\frac{1}{2 \lambda} \operatorname{Re}\left(-2 \bar{p} \cdot B\left(q, A^{-1} B(q, q)\right)+\bar{p} \cdot B\left(\bar{q},(2 \lambda i I-A)^{-1} B(q, q)\right)\right)=\frac{46}{815}$

which is positive. This ends the proof of the theorem.

\section{ACKNOWLEDGEMENTS}

The author is partially supported by a MINECO grant MTM2013-40998$\mathrm{P}$, an AGAUR grant number 2014SGR-568, the grants FP7-PEOPLE-2012IRSES 318999 and 316338, and the MINECO/FEDER grant UNAB13-4E1604. The second author is partially supported by FCT/Portugal through UID/MAT/04459/2013.

\section{REFERENCES}

[1] M. Abramowitz And I.A. Stegun, Elliptic Integrals, Ch. 17 in Handbook of Mathematical Functions with Formulas, Graphs, and Mathematical Tables, 9th printing. New York: Dover, pp. 587-607, 1972.

[2] C. Christopher, J. Llibre And J.V. Pereira, Multiplicity of invariant algebraic curves in polynomial vector fields, Pacific J. Math. 229 (2007), 63-117.

[3] A. Cima And J. Llibre, Bounded polynomial systems, Trans. Amer. Math. Soc. 318 (1990), 557-579.

[4] F. Dumortier, J. Llibre And J.C. Artes, Qualitative Theory of Planar Differential Systems, in: Universitext, Springer-Verlag, New York, 2006.

[5] Y. Kuznetsov, Elements of applied bifurcation theory, Applied Mathematical Sciences 112, Springer-Verlag, New York, 1998.

[6] J. Llibre And X. Zhang, Darboux theory of integrability in $\mathbb{C}^{n}$ taking into account the multiplicity, J. Differential Equations 246 (2009), 541-551.

[7] J. Llibre And X. Zhang, Darboux theory of integrability for polynomial vector fields in $\mathbb{R}^{n}$ taking into account the multiplicity at infinity, Bu.. Sci. Math. 133 (2009), 765-778.

[8] J.C. Sprott, X. Wang And G. Chen, Coexistence of point, periodic and strange attractors, Int. J. Bifurcation and Chaos 23 (2013), 1350093 (5 pp). 
${ }^{1}$ Departament de Matemàtiques, Universitat Autònoma de Barcelona, 08193 Bellaterra, Barcelona, Catalonia, Spain

E-mail address: jllibre@mat.uab.cat

Departamento de Matemática, Instituto Superior Técnico, Universidade de LisboA, 1049-001 LisboA, Portugal

E-mail address: cvalls@math.ist.utl.pt 